\title{
Detecção fenotípica de metalobetalactamase em isolados clinicos de Pseudomonas aeruginosa de hospitais de Caxias do Sul
}

\author{
Detection of phenotypic metallo-beta-lactamase in clinical isolates of Pseudomonas aeruginosa from hospitals \\ in Caxias do Sul
}

Franciele Maria Zanol'; Simone Ulrich Picoli²; Fernanda Morsch ${ }^{3}$

\section{unitermos \\ Pseudomonas aeruginosa \\ Metalobetalactamase \\ Resistência bacteriana}

\section{resumo}

Introdução: Entre os principais microrganismos causadores de infecções nosocomiais destacam-se bacilos Gram-negativos não fermentadores de glicose, como Pseudomonas aeruginosa. O desenvolvimento de mecanismos de resistência por estes patógenos, como a produção de metalobetalactamases (MBL), causa grande preocupação no âmbito hospitalar. Objetivo: Determinar a prevalência do fenótipo MBL em amostras de $P$. aeruginosa isoladas de hospitais de Caxias do Sul (RS) e comparar alguns métodos fenotípicos empregados para a detecção dessa enzima. Material e métodos: Um total de 50 amostras de $P$. aeruginosa com sensibilidade diminuída à ceftazidima foi submetido à avaliação fenotípica quanto à produção de $\mathrm{MBL}$ por meio de três testes: aproximação de discos, disco combinado e E-test $\mathrm{MBL}$, empregando diferentes substratos e inibidores da metaloenzima. Resultados: A produção de MBL foi verificada em 15 amostras (30\%). O método que apresentou maior percentual de resultados positivos foi o E-test MBL, seguido do teste de disco combinado de ceftazidima com ácido etilenodiaminotetracético (EDTA). Conclusão: A presença de MBL é um importante mecanismo de resistência encontrado nos hospitais participantes do estudo, demonstrando a necessidade da detecção dessas enzimas a fim de controlar a disseminação dos genes de resistência e proporcionar terapia adequada ao paciente.

\section{abstract}

Introduction: Non-fermenting Gram-negative bacilli, such as Pseudomonas aeruginosa, feature among the main microorganisms that cause nosocomial infections. The development of resistance mechanisms, such as the production of metallo-beta-lactamases (MBL), is a major concern in hospitals. Objectives: To determine the prevalence of MBL phenotype in samples of P. aeruginosa isolates from hospitals in Caxias do Sul (RS) and compare some phenotypic methods applied in this enzyme detection. Material and methods: A total of 50 samples of P. aeruginosa with decreased susceptibility to ceftazidime were phenotypically evaluated as to $M B L$ production by three tests: double-disk synergy, combined disk and MBL E-test, using different substrates and metallo-enzyme inhibitors. Results: The production of MBL was detected in 15 samples (30\%). The method showing higher percentage of positive results was the MBL E-test, followed by ceftazidime-EDTA disk method. Conclusion: The presence of MBL is an important resistance mechanism found in the studied hospitals, which substantiate the need for the detection of these enzymes in order to control the spread of resistance genes and provide appropriate therapy to the patient. key words

Pseudomonas aeruginosa

Metallo-beta-lactamase

Bacterial resistance

\footnotetext{
1. Bacharel em Biomedicina.

2. Mestra em Microbiologia; professora adjunta da Federação de Estabelecimento de Ensino Superior em Novo Hamburgo (FEEVALE).

3. Especialista em Microbiologia; bioquímica chefe do Laboratório de Microbiologia do Hospital Nossa Senhora Medianeira.
} 


\section{Introdução}

O surgimento de bactérias resistentes pode ser observado desde a introdução, na prática clínica, dos primeiros agentes antimicrobianos. No entanto, a rápida disseminação destes patógenos multirresistentes pode representar séria ameaça, considerando-se fatores já existentes, como as altas taxas de mortalidade, a restrição de tratamentos e a alta prevalência de bactérias resistentes a antimicrobianos no ambiente hospitalar(22).

Entre os principais microrganismos causadores de infecções nosocomiais e que apresentam importantes mecanismos de resistência destacam-se os bacilos Gramnegativos oportunistas, não fermentadores de glicose, como Pseudomonas aeruginosa ${ }^{(13,15)}$.

Um importante mecanismo de resistência encontrado em cepas desta bactéria é a produção de metalobetalactamases (MBLs). Trata-se de enzima capaz de hidrolisar o anel betalactâmico por meio de hidroxilação irreversível do grupo amida, causando inativação de antimicrobianos que incluem penicilinas, cefalosporinas e carbapenens, excetuando-se o aztreonam. Atuam também sobre os inibidores de serinobetalactamases, como ácido clavulânico, sulbactama e tazobactama ${ }^{(8)}$. O tratamento se restringe apenas a polimixina B ou colistina ${ }^{(19,22)}$.

Os genes que codificam MBLs estão localizados em plasmídeos, elementos móveis que conferem mobilidade ao gene e asseguram sua rápida disseminação, fato que gera grande preocupação(16).

Não existem, até o presente momento, testes fenotípicos ou moleculares para detecção de MBLs padronizados pelo Clinical and Laboratory Standards Institute $(\mathrm{CLSI})^{(9,17)}$. No entanto, conhecendo a crescente disseminação dos genes que codificam MBLs e os problemas que esses genes de resistência representam, vários estudos vêm sendo realizados a fim de desenvolver testes práticos, eficazes e economicamente viáveis para a deteç̧ão de amostras produtoras de metaloenzimas.

\section{Objetivos}

Determinar a prevalência do fenótipo de resistência de MBLs em cepas de $P$. aeruginosa isoladas de amostras clínicas de hospitais de Caxias do Sul (RS) e comparar alguns métodos fenotípicos empregados na pesquisa de metaloenzimas.

\section{Material e métodos}

\section{Amostras bacterianas}

Este estudo incluiu 50 cepas de $P$. aeruginosa isoladas de diferentes materiais clínicos e de diferentes pacientes provenientes de hospitais de Caxias do Sul no período de janeiro a outubro de 2009, sendo selecionadas amostras com sensibilidade diminuída a cefalosporinas de espectro ampliado. As amostras foram preservadas por congelamento a $-20^{\circ} \mathrm{C}$ em caldo glicerinado até a realização das pesquisas fenotípicas.

\section{Identificação das amostras}

Foram realizados testes a fim de confirmar a identidade das bactérias requeridas para o estudo, sendo descartadas as que não apresentavam características de $P$. aeruginosa.

A identificação bacteriana avaliou aspectos como odor característico, produção de pigmento, observação de características com a coloração de Gram, citrato positivo, não fermentação de glicose, crescimento a $42^{\circ} \mathrm{C}$, motilidade e oxidase positiva.

\section{Suscetibilidade aos antimicrobianos e pesquisa fenotípica para MBL}

Os discos de antimicrobianos testados foram ceftazidima (CAZ) $30 \mu \mathrm{g}$, imipenem (IPM) $10 \mu \mathrm{g}$ e aztreonam (ATM) $30 \mu \mathrm{g}$ (Biorad). As amostras que apresentaram sensibilidade reduzida à ceftazidima foram avaliadas quanto à produção de metalobetalactamase por meio de três metodologias que incluíram disco aproximação, disco combinado e E-test MBL.

Para a realização de todos os testes fenotípicos foram preparados inóculos bacterianos por suspensão direta em soro fisiológico ( $\mathrm{NaCl}$ a 0,9\%) a partir de colônias isoladas com 24 horas de crescimento. A turvação da suspensão foi ajustada à escala 0,5 de McFarland por espectofotômetro (625 nm). Os inóculos bacterianos foram semeados em placas de ágar Müeller-Hinton (MCBarth).

O teste de aproximação de discos foi realizado segundo Arakawa et al.(1). Um disco de CAZ $30 \mu \mathrm{g}$ foi posicionado a $10 \mathrm{~mm}$ do disco de papel-filtro com $3 \mu \mathrm{l}$ de ácido 2-mercaptopropiônico (2-MPA). Outro teste semelhante foi realizado utilizando-se ácido etilenodiaminotetracético (EDTA) como inibidor de MBL. Foram adicionados $5 \mu \mathrm{lde}$ solução EDTA 0,5 $\mathrm{M}$ ao disco de papel-filtro posicionado a uma distância de $10 \mathrm{~mm}$ de disco de IPM $10 \mu \mathrm{g}$. A observação de uma ampliação na forma do halo de inibição do 
antimicrobiano onde houve a difusão do agente quelante (EDTA ou 2-MPA) indicou amostra positiva para MBL. Em testes negativos para a produção dessa enzima, alterações no halo de inibição do crescimento da bactéria testada não foram observadas.

A segunda metodologia utilizada foi o disco combinado de substratos com e sem EDTA. Este teste consistiu na colocação de dois discos de um mesmo substrato (CAZ ou IPM) no ágar e a um deles foram adicionados $10 \mu \mathrm{l}$ de EDTA $(0,5 \mathrm{M}$ ou $750 \mu \mathrm{g})$. Para interpretação, os halos de substrato com e sem EDTA foram comparados e o achado de diferença maior ou igual a $7 \mathrm{~mm}$ no halo de substrato/ EDTA em relação ao substrato sozinho foi considerado positivo para $\mathrm{MBL}^{(14,17,23)}$.

Para completar os testes realizados foram utilizadas fitas de E-test MBL (AB Biodisk, Dalvägen, Solna, Sweden), compostas por IPM em uma das extremidades e IPM associado ao EDTA em outra extremidade. Nesta metodologia foram consideradas amostras positivas aquelas que apresentaram concentração inibitória mínima (MIC) de IPM/EDTA três diluições abaixo daquela demonstrada pela amostra em presença apenas de $\operatorname{IPM}^{(14)}$.
O controle de qualidade dos testes fenotípicos para $M B L$ foi realizado por meio da utilização da cepa de P. aeruginosa positiva (SPM-1) cedida pelo Hospital das Clínicas da faculdade de Medicina da Universidade de São Paulo (HCFMUSP). Para o controle negativo foi utilizada a cepa de $P$. aeruginosa ATCC 27853.

\section{Resultados}

A presença da enzima MBL foi verificada em 15 (30\%) amostras de $P$. aeruginosa, sendo duas (13\%) amostras MBL detectadas em cinco dos seis testes utilizados e outras cinco (33\%) em quatro testes (Tabela 1).

O teste que apresentou maior percentual para resultados positivos foi a metodologia de E-test MBL (100\%), seguido pelo disco combinado de CAZ/EDTA 0,5 M (86,7\%). Por outro lado, o teste de disco combinado de IPM/EDTA $750 \mathrm{\mu g}$ forneceu resultados negativos para todas as amostras, inclusive para o controle positivo (SPM-1) (Tabela 1). A Tabela 2 demonstra o percentual de resultados positivos e negativos encontrados para cada teste realizado.

\section{Tabela 1}

Resultados dos testes para deteç̧ão de MBL em amostras de $P$. aeruginosa, de acordo com cada

\begin{tabular}{|c|c|c|c|c|c|c|}
\hline Amostra & $\begin{array}{c}\text { E-Test IPM/ } \\
\text { EDTA }\end{array}$ & $\begin{array}{c}\text { Aprox. } \\
\text { CAZ/2-MPA }\end{array}$ & $\begin{array}{c}\text { Aprox. IMP/EDTA } \\
0,5 \mathrm{M}\end{array}$ & $\begin{array}{l}\text { Comb. IMP/ } \\
\text { EDTA 0,5 M }\end{array}$ & $\begin{array}{l}\text { Comb. CAZI } \\
\text { EDTA 0,5 M }\end{array}$ & $\begin{array}{c}\text { Comb. IMP/EDTA } \\
750 \mu \mathrm{g}\end{array}$ \\
\hline A & + & - & - & - & + & - \\
\hline B & + & - & - & + & - & - \\
\hline C & + & - & - & - & + & - \\
\hline D & + & + & + & - & + & - \\
\hline E & + & + & - & - & + & - \\
\hline $\mathrm{F}$ & + & + & + & + & + & - \\
\hline G & + & + & + & - & + & - \\
\hline $\mathrm{H}$ & + & + & - & - & - & - \\
\hline I & + & + & + & + & + & - \\
\hline J & + & + & + & - & + & - \\
\hline K & + & - & - & - & + & - \\
\hline L & + & - & - & - & + & - \\
\hline M & + & - & - & + & + & - \\
\hline $\mathrm{N}$ & + & - & + & + & + & - \\
\hline 0 & + & + & + & - & + & - \\
\hline SPM-1 & + & + & + & + & + & - \\
\hline ATCC & - & - & - & - & - & - \\
\hline
\end{tabular}

(+): positivo; (-): negativo; Aprox.: aproximação de discos; COMB.: disco combinado; SPM-1: São Paulo metalobetalactamase; ATCC: P. aeruginosa 27853 negativa para $M B L$. 
Tabela 2 Percentual dos resultados positivos e negativos em cada teste realizado

\begin{tabular}{l|c|c|c|c}
\multicolumn{1}{c}{ Resultado } & \multicolumn{2}{c}{ Positivo } & \multicolumn{2}{c}{ Negativo } \\
\multicolumn{1}{c|}{ Teste } & N & $\%$ & N & $\%$ \\
\hline E-test & 15 & 100 & 0 & 46,7 \\
CAZ/2-MPA (Aprox.) & 8 & 53,3 & 7 & 53,3 \\
IPM/EDTA 0,5 M (Aprox.) & 7 & 46,7 & 8 & 66,7 \\
IPM/EDTA 0,5 M (Comb.) & 5 & 33,3 & 10 & 13,3 \\
CAZ/EDTA 0,5 M (Comb.) & 13 & 86,7 & 2 & 100 \\
\hline IPM/EDTA 750 $\mu$ g (Comb.) & 0 & 0 & 15 & 0 \\
\hline
\end{tabular}

Aprox.: aproximação de discos; Comb.: disco combinado.

A maior parte das amostras MBL positivas foram provenientes de secreção traqueal e escarro, correspondendo a $46 \%$ e $34 \%$, respectivamente. As demais amostras com metaloenzima foram isoladas de urina (14\%) e de outros materiais biológicos (6\%), como sangue e secreção de feridas (queimaduras).

\section{Discussão}

A produção de $\mathrm{MBL}$ em $P$. aeruginosa está se tornando cada vez mais frequente no $\mathrm{Brasil}^{(7)}$. Neste estudo, $30 \%$ das amostras desta bactéria apresentaram teste fenotípico positivo para $\mathrm{MBL}$, demonstrando que se trata de uma enzima com grande capacidade de disseminação e frequente no âmbito hospitalar ${ }^{(14,18)}$.

Os dados de frequência de metaloenzimas em nível mundial são muito diversificados: na Itália já foi descrita taxa de $39,1 \%{ }^{(10)}$, enquanto na China este índice ultrapassou $65 \%{ }^{(5)}$. No Brasil, o panorama também é de grande oscilação, com relatos de aproximadamente $20 \%^{(7)}$ até mais que $75 \%{ }^{(25)}$.

Este estudo avaliou algumas das principais metodologias descritas para detecção fenotípica de MBL. São testes rápidos, simples e de baixo custo, no entanto os resultados obtidos podem sofrer variações de acordo com a bactéria testada, a enzima pesquisada, o substrato e o agente quelante utilizado ${ }^{(14)}$.

Embora a metodologia envolvendo E-test MBL tenha apresentado maior percentual de resultados positivos (100\%) e, aparentemente, corresponda ao melhor teste para detecção de $\mathrm{MBL}^{(20)}$, foi possível observar que o método de disco combinado de IPM/EDTA, com base no mesmo princípio das fitas de E-test MBL, não apresentou resultados equivalentes. Este método testou duas concentrações diferentes de EDTA $(0,5 \mathrm{M}$ e $750 \mu \mathrm{g})$ e os resultados observados para ambas as concentrações testadas não foram satisfatórios quando em comparação com os obtidos por outras metodologias.

$O$ teste de disco combinado de IPM/EDTA $750 \mu \mathrm{g}$ falhou na detecção de todas as amostras com resultados positivos em outros métodos, inclusive na amostra utilizada como controle positivo. Este resultado confronta outros dados obtidos em estudo no qual se encontrou elevadas especificidade e sensibilidade na detecção de cepas de $\mathrm{MBL}^{(12)}$. Um estudo realizado em 2002 pelo próprio autor da técnica demonstrou que esta metodologia apresentou $4 \%$ de resultados falso negativos quando em comparação com a reação em cadeia de polimerase (PCR) ${ }^{(23)}$.

É importante ressaltar que o E-test MBL é um método quantitativo e de fácil interpretação, contudo pode fornecer resultados falso positivos, pois pode superestimar a presença de MBL devido ao aumento da permeabilidade da parede celular e, pelo zinco quelado pelo EDTA, acelerar a decomposição do imipenem e diminuir a expressão de OprD de $P$. aeruginosa ${ }^{(2)}$. Por isso se recomenda cautela na interpretação dos resultados de testes com EDTA ${ }^{(3)}$.

Por outro lado, o uso de ceftazidima e EDTA 0,5 M no teste de disco combinado apresentou bons resultados, detectando $86,7 \%$ das cepas MBL. Um aspecto que merece destaque é a facilidade na interpretação destes testes, pois é conduzida mediante medida de diâmetros dos halos de inibição, isentando o analista de erros subjetivos como a "ampliação/distorção do halo".

A terceira metodologia que apresentou maior percentual de resultados positivos foi o teste de disco aproximação $(53,3 \%)$, utilizando como agente quelante o 2-MPA. Existe a possibilidade de este dado ter sido subestimado, considerando-se o caráter de subjetividade inerente à interpretação do mesmo. 
Vários estudos comparando substratos e agentes quelantes utilizados na detecção fenotípica de MBL já foram realizados. Em um dos primeiros publicados sobre métodos para detecção fenotípica de $\mathrm{MBL}$, três agentes quelantes foram empregados: o 2-MPA, marcaptoetanol e EDTA (CAZ como substrato), tendo sido comprovado que o 2-MPA apresentou maior sensibilidade (100\%) ante os demais agentes quelantes ${ }^{(1)}$. Outra pesquisa realizada também demonstrou bons resultados nos testes de disco aproximação utilizando $3 \mu \mathrm{l}$ de 2-MPA e CAZ ${ }^{(11)}$. Um trabalho brasileiro comparando os métodos de aproximação de discos e discos combinados utilizando diferentes inibidores de MBL, em isolados de $P$. aeruginosa produtores de SPM-1, demonstrou que a melhor técnica seria a de disco aproximação utilizando ceftazidima e 2-MPA ${ }^{(16)}$, corroborando Arakawa et al. ${ }^{(1)}$ e Lee et al..$^{(11)}$.

Outros autores confirmaram, em pesquisas comparando métodos fenotípicos, que o EDTA seria o agente quelante mais eficiente para detecção de microrganismos produtores de metalobetalactamase ${ }^{(13,17,19)}$. Em nosso estudo, este foi o agente quelante utilizado em duas metodologias que apresentaram os melhores percentuais de resultados positivos (E-test MBL e CAZ/EDTA 0,5 M).

Considerando que os testes CAZ/EDTA $0,5 \mathrm{M}$ e CAZ/2MPA foram conduzidos com o mesmo substrato (CAZ), mas com inibidores de MBL diferentes, enfatiza-se a possibilidade de interpretação incorreta naquele em que não é possível a "mensuração" do resultado (CAZ/2-MPA).

Uma possível explicação para as discrepâncias nos resultados encontrados é a grande variabilidade genética destas enzimas. Um estudo realizado aplicou a técnica de PCR em amostras isoladas de cinco estados brasileiros que apresentaram resultados positivos para $\mathrm{MBL}$ nos testes fenotípicos e identificou a presença da família SPM, encontrada somente no Brasil(7, 21).

Alguns estudos comparando métodos de detecção de MBL já foram realizados, demonstrando que os testes fenotípicos apresentam boas sensibilidade e especificidade. No entanto, os resultados obtidos podem sofrer variações de acordo com a bactéria testada, o tipo de enzima pesquisada, o substrato e $o$ agente quelante utilizado ${ }^{(14)}$.

Os resultados obtidos neste trabalho demonstram que a utilização simultânea de várias metodologias seria a decisão mais coerente para a obtenção de resultados seguros e precisos na detecção de microrganismos produtores de MBL, já que não existe combinação ideal de inibidores de metaloenzimas e agentes betalactâmicos capaz de detectar, em um único teste, todas as famílias de MBL existentes ${ }^{(6,11,13)}$.

Uma deficiência desta pesquisa foi a impossibilidade de realizar estudos moleculares a fim de caracterizar e confirmar os determinantes genéticos envolvidos. Por esta razão, todas as amostras com fenótipo de MBL serão posteriormente submetidas a estudos adicionais para melhor compreensão dos resultados.

\section{Conclusão}

Este estudo encontrou taxas elevadas de $P$. aeruginosa produtoras de $\mathrm{MBL}$, reforçando a necessidade de medidas eficazes por parte da Comissão de Controle de Infecção Hospitalar dos hospitais incluídos no estudo, a fim de conter a rápida disseminação destas enzimas e restringir o uso de antimicrobianos, especialmente carbapenêmicos.

O uso de testes fenotípicos para detecção de metaloenzimas se torna de extrema relevância no diagnóstico de microrganismos produtores de MBL, principalmente quando várias metodologias são utilizadas concomitantemente. O E-test MBL e o disco combinado de ceftazidima com EDTA demonstraram, neste estudo, serem testes com boa sensibilidade e de fáceis execução e interpretação na detecção de metaloenzimas na rotina laboratorial.

\section{Referências}

1. ARAKAWA, Y. et al. Convenient test for screening metallo$\beta$-lactamase-producing Gram-negative bacteria by using thiol compounds. J Clin Microbiol, v. 38, n. 1, p. 40-3, 2000.

2. CORNEJO, M. C. et al. Zinc eluted from siliconized latex urinary catheters decreases OprD expression, causing carbapenem resistance in Pseudomonas aeruginosa. Antimicrob Agents Chemoter, v. 47, n. 7, p. 2313-5, 2003.
3. DANEL, F. et al. Effect of divalent metal cations on the dimerization of OXA-10 and -14 class D $\beta$-lactamases from Pseudomonas aeruginosa. Biochemistry, v. 40, p. 9412-20, 2001.

4. DOLARA, P. et al. EDTA susceptibility leading to false detection of metallo- $\beta$-lactamase in Pseudomonas aeruginosa by Etest and an imipinem-EDTA disk method. Internat J Antimicrob Agents, v. 26, n. 4, p. 340-1, 2006. 
5. DONG, F. et al. Characterization of multidrug-resistance and metallo-beta-lactamase-proucing Pseudomonas aeruginosa isolates from a paediatric clinic in China. Chines Medic J, v. 121, n. 17, p. 340-1, 2008.

6. FRANKLIN, C. et al. Phenotypic detection of carbapenemsusceptible metallo- $\beta$-lactamases-producing Gramnegative bacilli in the clinical laboratory. J Clin Microbiol, v. 44, n. 3, p. 3139-44, 2006.

7. GALLES, A.C et al. Dissemination in distinct Brazilian regions of an epidemic carbapenem-resistant Pseudomonas aeruginosa producing SPM mettalo- $\beta$-lactamase. J Antimicrob Chemother, v. 52, n. 4, p. 699-702, 2003.

8. GUPTA. V. Metallo beta lactamase in Pseudomonas aeruginosa and Acinetobacter species. Expert Opin Investing Drugs, v. 17, n. 2, p. 131-43, 2008.

9. JESUDASON, M. V. et al. Comparison of two methods to detect carbapenemase e metallo- $\beta$-lactamase production in clinical isolates. Ind J Med Res, v. 121, n. 6, p. 780-83, 2005.

10. JONES, R. N. et al. Emerging epidemic of metallo$\beta$-lactamase-mediated resistances. Diagn Microbiol Infect Dis, v. 51, p. 77-84, 2005.

11. LEE, K. et al. Modified Hodge and EDTA-disk synergy tests to screen metallo- $\beta$-lactamase-producing strains of Pseudomonas and Acinetobacter species. Clin Microbiol Infect, v. 7, p. 88-91, 2001.

12. MAGALHÃES, V. et al. Metallo- $\beta$-lactamase producing Pseudomonas aeruginosa strains isolated in hospital in Recife, PE, Brazil. Brazilian J Microbiol, v. 36, p. 123-5, 2005.

13. MARRA, A. R. et al. Bloodstream infections with metallobeta-lactamase-producing Pseudomonas aeruginosa: epidemiology, microbiology and clinical outcomes. Antimicrob Agents Chemother, v. 50, n. 1, p. 388-90, 2006.

14. MENDES, R. E. et al. Metallo- $\beta$-Lactamase. J Bras Ptol Med Lab, v. 42, n. 2, p. 103-13, 2006.

15. PATERSON, D. L. The epidemiological profile of infections with multidrug-resistant Pseudomonas aeruginosa and Acinetobacter species. Clin Infect Dis, v. 43, n. 1, p. 43-8, 2006.
16. PICÃO, R. C. et al. Metallo- $\beta$-lactamse detection: comparative evaluation of double-disk synergy versus combined disk tests for IMP-, GIM-, SIM-, SPM-, or VIM-producing isolates. J Clin Microbiol, v. 46, n. 6, p. 2028-37, 2006.

17. PITOUT, J. D. D. et al. Detection of Pseudomonas aeruginosa producing metallo-beta-lactamase in a large centralized laboratory. J Clin Microbiol, v. 43, n. 7, p. 3129-35, 2005.

18. SADER, H. S. et al. IMPs, VIMs and SPMs: the diversity of metallo- $\beta$-lactamases produced by carbapenemresistant pseudomonas aeruginosa in a Brazilian hospital. Clin Microb Infect, v. 11, p. 73-9, 2005.

19. TAN, J. et al. News and sensitive assay for determining Pseudomonas aeruginosa metallo-beta-lactamase resistance to imipinem. J Clin Microbiol, v. 46, n. 5, p. 1870-72, 2008.

20. TING-TIN, Q. et al. Evaluation of phenotypic tests for detection of metallo-beta-lactamase-producing Pseudomonas aeruginosa strains in China. J Clin Microbiol, v. 47, n. 4, p. 1136-42, 2009.

21. TOLEMAN, M. A. et al. Molecular characterization of SPM-1, a novel metallo- $\beta$-lactamase isolated in Latin America: report from the SENTRY Antimicrobial Surveillance Program. J Antimicrob Chemother, v. 50, n. 5, p. 673-9, 2002.

22. WALSH, T. R. et al. Metallo- $\beta$-lactamases: the quiet before the storm? Clin Microbiol Rev, v. 18, n. 2, p. 306-25, 2005.

23. YAN. J. J. et al. Comparison of the double-disk, combined disk, and Etest methods for detecting metallo-betalactamases in Gram-negative bacilli. Diagn Microbiol Infect Dis, v. 49, n. 1, p. 5-11, 2004.

24. YONG, D. et al. Imipinem-EDTA disk method for differentiation of metallo- $\beta$-lactamase-producing clinical isolates of Pseudomonas sp. and Acinetobacter sp. J Clin Microbiol, v. 40, n. 10, p. 3789-801, 2002.

25. ZAVASCKY, A. P. et al. Polymyxin B for the treatment of multidrug-resistant pathogens: a critical review. J Antimicrob Chemother, v. 60, p. 1206-15, 2007. 